Experiencia educativa

\title{
Centro de escritura en la Escuela de Idiomas de la Universidad Don Bosco
}

\author{
The Writing center at the School of Languages at Universidad Don Bosco \\ Lorena Beatriz Pérez de Cabrera* \\ lorena.perez@udb.edu.sv
}

ISSN 1996-1642, Editorial Universidad Don Bosco, año 8, No.13, Enero-Junio de 2014, pp. 33-40

Recibido: 29 de noviembre de 2013. Aprobado: 6 de enero de 2014

\section{Resumen}

Este artículo narra la experiencia educativa exitosa de la implementación de un Centro de Escritura en la Escuela de Idiomas de la Universidad Don Bosco enEl Salvador durante el ciclo uno del año 2013. Primeramente, se examina el concepto de escritura académica por parte de los docentes de idiomas y como este concepto, por su naturaleza, no facilita el desarrollo de esta habilidad. A continuación se relata cómo el Centro de Escritura se integró como un proyecto de la cátedra Composición en Inglés 2 y brindó más de 100 asesorías a estudiantes de diversas asignaturas en inglés y español. Finalmente, la experiencia permite señalar aspectos puntuales que de ser apoyados se trasladarían en una mejora significativa de los niveles de alfabetización académica de los estudiantes de idiomas en la UDB.

Palabras clave: escritura académica, alfabetización académica, centro de escritura, habilidades

\section{Abstract}

This paper narrates the successful educational experience of opening a Writing Center at the School of Languages at Universidad Don Bosco (UDB) in El Salvador during the semester 01- 2013. First, the paper approaches the concept of academic writing by language teachers and how this concept, by its nature, does not facilitate the development of this skill. Then, the author explains how the Writing Center was integrated as a course project of English Composition 2 providing over 100 consulting sessions to students in various subjects in English and Spanish. Finally, based on this experience, specific aspects are pointed out, which should be supported to promote a significant improvement in the language students' academic literacy levels at the UDB.

Keywords: academic writing, academic literacy, writing center, skills

* Profesora de la Escuela de Idiomas de la Universidad Don, Bosco y Máster en enseñanza del idioma Inglés a hablantes de otros idiomas por la Universidad de Michigan, Estados Unidos.

Para citar este artículo: Pérez de Cabrera, L. B. (2014). Centro de escritura en la Escuela de Idiomas de la Universidad Don Bosco. Diá-logos 13, 33-40. 


\section{Introducción}

La escritura, junto con la comprensión auditiva, la producción oral y la lectura, es una de las cuatro macro-habilidades lingüísticas a desarrollar en el proceso de aprendizaje de un idioma extranjero. Para muchos estudiantes de inglés como idioma extranjero (EFL por sus siglas en inglés), la escritura representa una de las habilidades más difíciles de desarrollar. Este sentir es comprensible al darse cuenta de que escribir es una habilidad compleja y, más aún, cuando se analiza los desafíos de escribir en un idioma que no es el propio. Aunado a este hecho, también en crucial reconocer que no se trata de producir cualquier tipo de escritura, sino más bien de escribir en el contexto académico universitario el cual es sumamente complejo y desafiante. Es a esta combinación, idioma extranjero-escritura académica, a la que los estudiantes de idiomas en las universidades latinoamericanas se enfrentan, y la Universidad Don Bosco (UDB), en El Salvador, no es la excepción. Estos factores animaron a la autora a impulsar el proyecto Centro de Escritura en la UDB durante el primer semestre del año 2013. El proyecto del Centro tenía dos propósitos primordiales. Primero, lograr que los estudiantes cursando Composición en inglés 2 obtuvieran una mayor compresión de cómo funciona la escritura académica en inglés y de cómo escribir es un acto comunicativo que transmite ideas las cuales se transforman dependiendo del mensaje a transferir. Segundo, y para lograr tal comprensión, los estudiantes de Composición fueron entrenados para brindar asesorías a estudiantes escritores de otras materias, cumpliendo así el primer propósito y expandiendo el beneficio a otros escritores universitarios. Este artículo brinda una narración de la experiencia de la cual surgen reflexiones significativas en miras a innovar las ideas ya concebidas sobre escribir académicamente.

\section{Escribir también es comunicar}

Muchos concordarán que la palabra escrita tiene tanto o más poder como lo que se expresa verbalmente. Sin embargo, en los contextos académicos latinoamericanos, escribir está frecuentemente relacionado a evaluaciones, exámenes y calificaciones. Esta es la manera de concebir la escritura para la gran mayoría de profesores y, en consecuencia, para la gran mayoría de estudiantes. Un escrito está bien sí, y solo sí, no contiene ni errores gramaticales, ni ortográficos ni de puntuación y transmite eficaz y fluidamente un mensaje. Tal es el caso de las tareas de escritura en la Escuela de Idiomas en la UDB. En todos los cursos de inglés o francés comunicativo, gramática e incluso en composición se pide a los estudiantes que escriban ' $x$ ' cantidad de palabras sobre ' $y$ ' tema y que utilicen ' $z$ ' estructuras del idioma inglés o francés. Por ejemplo, la Tabla 1, presentada a continuación, es la asignación de una evaluación de escritura 
el cual escribir y la rúbrica utilizada para calificar el escrito. De este ejemplo se puede observar los siguientes aspectos: no existe un proceso de escritura', el tema del cual se debe escribir es meramente personal en vez de académico/ profesional y el énfasis en aspectos de mecánica y forma, aunque importantes, opaca el propósito comunicativo de escribir. La escritura concebida de tal modo, adquiere una dimensión únicamente remedial que la aleja del propósito comunicativo del cual originalmente parte cualquier iniciativa de escritura. No se trata de demeritar la forma del idioma, sino más bien de no perder de vista por qué se escribe.

En contraste, cuando se vincula la escritura a los contextos académicos y a la realidad profesional de los estudiantes, es posible considerar que lo que se escribe esta de algún modo comunicando un sentir, una opinión, una crítica, etc. sobre cualquier tema en estudio. Scardamalia y Bereiter (citado en Molina Natera, 2012) afirman al respecto, "En cuanto a la escritura, es tomada como un instrumento de evaluación con el que los estudiantes 'dicen el conocimiento', en lugar de transformarlo considerando la situación retórica (quién es el lector y qué se quiere lograr en él) y el contenido mismo" (p. 95). En otras palabras, la escritura no debería desligarse de su propósito primordial el cual es comunicar ideas propias, es decir, crear conocimiento.

Un primer paso para lograr este propósito es el permitir a los estudiantes atravesar el proceso de escritura. Todo escritor profesional sabe que para obtener un producto final, se necesita de muchos borradores, revisiones y correcciones que al final se convertirán en su propia voz ante sus lectores. Es imprescindible otorgar esta misma oportunidad a los estudiantes que principian el desarrollo de sus habilidades de escritura. Un segundo paso es el que señala Carlino (2012) al decir que "no se aprende a escribir en el vacío sino a partir del campo de problemas inherentes a una determinada disciplina" (p. 414). Es decir, que no se trata de pedirles a los estudiantes que escriban sobre cuestiones meramente personales cuando ellos mismos son profesionales en desarrollo de las áreas turísticas y de enseñanza, para el caso de la Escuela de Idiomas en la UDB. La combinación del proceso de escritura y un tema relevante al campo de estudio son factores claves para lograr transformar la perspectiva de escribir para evaluar a escribir para comunicar.

1 Se entiende por proceso de escritura aquel que permite desarrollar un escrito por etapas, es decir, pre-writing, writing y editing (Ryan y Zimmerelli, 2010). 
Tabla 1. Muestra de evaluación escrita de período para Gramática del inglés 2

Write a paragraph for each of the different situations given

\section{UNIT 15 TASK/ GOING ON TRIP}

One of your best friends is going on a trip to a country he's never been to. Your task will be to advise him what to take with him and what not to do in a country he doesn't know. You can also give urgent advice and express necessity on the things he's not supposed to forget.

\begin{tabular}{|c|c|c|c|c|c|c|}
\hline Conventions & $\begin{array}{c}\text { Failing } \\
0\end{array}$ & $\begin{array}{c}\text { Limited } \\
4\end{array}$ & $\begin{array}{l}\text { Emerging } \\
6\end{array}$ & $\begin{array}{c}\text { Competent } \\
8\end{array}$ & $\begin{array}{c}\text { Exemplary } \\
10\end{array}$ & Score \\
\hline $\begin{array}{c}\text { Correct use } \\
\text { of } \\
\text { sentence } \\
\text { structures } \\
40 \%\end{array}$ & $\begin{array}{l}\text {-Doesn't write } \\
\text { or misuses } \\
\text { the required } \\
\text { structures } \\
\text { studied in } \\
\text { class. } \\
\text {-Many } \\
\text { mistakes } \\
\text { that Interfere } \\
\text { with the } \\
\text { understanding } \\
\text { of the } \\
\text { message are } \\
\text { found. } \\
\text {-Needs } \\
\text { improvement. }\end{array}$ & $\begin{array}{l}\text {-Shows a poor } \\
\text { command } \\
\text { of most of } \\
\text { the direct } \\
\text { and indirect } \\
\text { speech tense } \\
\text { changes, } \\
\text { structure and } \\
\text { function. } \\
\text {-Sentences } \\
\text { are not } \\
\text { structured } \\
\text { according to } \\
\text { the standard } \\
\text { English word } \\
\text { order. } \\
\text { - Many } \\
\text { grammar } \\
\text { mistakes are } \\
\text { found. } \\
\text {-Uses simple } \\
\text { grammar } \\
\text { structures, } \\
\text { and makes } \\
\text { many errors. }\end{array}$ & $\begin{array}{l}\text { - Shows } \\
\text { average } \\
\text { command of } \\
\text { the direct and } \\
\text { indirect speech } \\
\text { tense changes, } \\
\text { structure and } \\
\text { function. } \\
\text { - Half of the } \\
\text { sentences } \\
\text { are structured } \\
\text { according to } \\
\text { the standard } \\
\text { English word } \\
\text { order. } \\
\text { - Some } \\
\text { grammar } \\
\text { mistakes are } \\
\text { found. } \\
\text {-Some verbs } \\
\text { are not } \\
\text { conjugated } \\
\text { correctly } \\
\text { (present, past, } \\
\text { past participle) }\end{array}$ & $\begin{array}{l}\text { - Shows good } \\
\text { command } \\
\text { of the direct } \\
\text { and indirect } \\
\text { speech tense } \\
\text { changes, } \\
\text { structure and } \\
\text { function. } \\
\text { - Most } \\
\text { sentences } \\
\text { are structured } \\
\text { according to } \\
\text { the standard } \\
\text { English word } \\
\text { order. } \\
\text { - Few } \\
\text { grammar } \\
\text { mistakes are } \\
\text { found. } \\
\text {-Most } \\
\text { verbs are } \\
\text { conjugated } \\
\text { correctly } \\
\text { (present, } \\
\text { past, past } \\
\text { participle) }\end{array}$ & $\begin{array}{l}\text {-Shows } \\
\text { accurate } \\
\text { command } \\
\text { of modals, } \\
\text { structure and } \\
\text { function. } \\
\text { - All } \\
\text { sentences } \\
\text { are structured } \\
\text { according to } \\
\text { the standard } \\
\text { English word } \\
\text { order. } \\
\text {-Grammar } \\
\text { mistakes are } \\
\text { not found. } \\
\text {-All verbs are } \\
\text { conjugated } \\
\text { correctly } \\
\text { (present, } \\
\text { past, past } \\
\text { participle) } \\
\text {-Correct use } \\
\text { of complex } \\
\text { sentence } \\
\text { types } \\
\text { according } \\
\text { to the level is } \\
\text { observed. }\end{array}$ & \\
\hline $\begin{array}{c}\text { Coherence } \\
\text { and } \\
\text { Cohesion } \\
20 \%\end{array}$ & $\begin{array}{l}\text { - Ideas } \\
\text { don't follow } \\
\text { each other } \\
\text { in a logical } \\
\text { sequence. } \\
\text {-ldeas aren't } \\
\text { developed. }\end{array}$ & $\begin{array}{l}\text {-Few ideas } \\
\text { follow each } \\
\text { other in } \\
\text { a logical } \\
\text { sequence. } \\
\text {-ldeas are } \\
\text { incomplete } \\
\text { and isolated. }\end{array}$ & $\begin{array}{l}\text {-Some ideas } \\
\text { follow each } \\
\text { other in } \\
\text { a logical } \\
\text { sequence } \\
\text { with few } \\
\text { transitions and } \\
\text { conjunctions. } \\
\text {-Incomplete } \\
\text { ideas make } \\
\text { difficult to } \\
\text { understand the } \\
\text { message in the } \\
\text { paragraph. }\end{array}$ & $\begin{array}{l}\text {-Most ideas } \\
\text { follow each } \\
\text { other in } \\
\text { a logical } \\
\text { sequence } \\
\text { with clear } \\
\text { transitions and } \\
\text { conjunctions. } \\
\text {-Some } \\
\text { incomplete } \\
\text { ideas or } \\
\text { sentences } \\
\text { make difficult } \\
\text { to understand } \\
\text { the message } \\
\text { in the } \\
\text { paragraph. }\end{array}$ & $\begin{array}{l}\text {-All ideas } \\
\text { follow each } \\
\text { other in } \\
\text { a logical } \\
\text { sequence } \\
\text { with clear } \\
\text { transitions } \\
\text { and } \\
\text { conjunctions. } \\
\text { - All ideas } \\
\text { are fully } \\
\text { developed } \\
\text { in the } \\
\text { paragraph. }\end{array}$ & \\
\hline
\end{tabular}

Centro de escritura en la Escuela de Idiomas de la Universidad Don 


\begin{tabular}{|c|c|c|c|c|c|}
\hline $\begin{array}{c}\text { Punctuation } \\
20 \%\end{array}$ & $\begin{array}{l}\text { - Many } \\
\text { punctuation } \\
\text { errors hinder } \\
\text { the message. } \\
\text {-No proper } \\
\text { capitalization. } \\
\text {-Periods, } \\
\text { commas, } \\
\text { quotation } \\
\text { marks and } \\
\text { semi colons } \\
\text { are misused. }\end{array}$ & $\begin{array}{l}\text { - Punctuation } \\
\text { errors interfere } \\
\text { to convey the } \\
\text { message. } \\
\text {-Most } \\
\text { sentences } \\
\text { show not } \\
\text { proper } \\
\text { capitalization. } \\
\text {-Most periods, } \\
\text { commas and } \\
\text { semi colons, } \\
\text { and quotation } \\
\text { marks are } \\
\text { misused. }\end{array}$ & $\begin{array}{l}\text { - Punctuation } \\
\text { errors barely } \\
\text { interfere to } \\
\text { convey the } \\
\text { message. } \\
\text {-Some } \\
\text { sentences } \\
\text { show proper } \\
\text { capitalization. } \\
\text {-Some periods, } \\
\text { commas, } \\
\text { semi colons, } \\
\text { and quotation } \\
\text { marks are } \\
\text { misused. }\end{array}$ & $\begin{array}{l}\text { - Punctuation } \\
\text { errors do not } \\
\text { interfere to } \\
\text { convey the } \\
\text { message. } \\
\text { Most } \\
\text { sentences } \\
\text { show proper } \\
\text { capitalization. } \\
\text {-Periods, } \\
\text { commas, } \\
\text { semi colons } \\
\text { and quotation } \\
\text { marks are } \\
\text { used when } \\
\text { necessary. }\end{array}$ & $\begin{array}{l}\text {-Good } \\
\text { command of } \\
\text { punctuation } \\
\text { makes it } \\
\text { easier to } \\
\text { understand } \\
\text { the message. } \\
\text { - All } \\
\text { sentences } \\
\text { show proper } \\
\text { capitalization. } \\
\text {-Periods, } \\
\text { commas, } \\
\text { semicolons } \\
\text { and } \\
\text { quotation } \\
\text { marks are } \\
\text { used when } \\
\text { necessary. }\end{array}$ \\
\hline $\begin{array}{c}\text { Vocabulary } \\
\text { and spelling } \\
20 \%\end{array}$ & $\begin{array}{l}\text {-Only basic } \\
\text { vocabulary } \\
\text { and } \\
\text { expressions are } \\
\text { used. } \\
\text {-Makes too } \\
\text { many } \\
\text { Mistakes in } \\
\text { word choice } \\
\text { and spelling. }\end{array}$ & $\begin{array}{l}\text { Uses only } \\
\text { basic } \\
\text { vocabulary } \\
\text { and } \\
\text { expressions. } \\
\text {-Makes many } \\
\text { mistakes in } \\
\text { word choice } \\
\text { and spelling. }\end{array}$ & $\begin{array}{l}\text {-Uses limited } \\
\text { vocabulary } \\
\text { and } \\
\text { expressions. } \\
\text {-Some mistakes } \\
\text { in word choice. } \\
\text { - Some words } \\
\text { are incorrectly } \\
\text { spelled. }\end{array}$ & $\begin{array}{l}\text {-Varies } \\
\text { vocabulary } \\
\text { and } \\
\text { expressions, } \\
\text { but makes } \\
\text { occasional } \\
\text { errors in word } \\
\text { choice. } \\
\text {-Most words } \\
\text { are correctly } \\
\text { spelled. }\end{array}$ & $\begin{array}{l}\text {-Uses a } \\
\text { variety of } \\
\text { vocabulary } \\
\text { and } \\
\text { idiomatic } \\
\text { expressions } \\
\text { related to } \\
\text { task. } \\
\text {-Words are } \\
\text { correctly } \\
\text { spelled. } \\
\text { - No mistakes } \\
\text { in word } \\
\text { choice. }\end{array}$ \\
\hline
\end{tabular}

\section{El Centro de Escritura en la UDB}

Después de compartir la idea de establecer un Centro de Escritura con el director de escuela, me fue asignado impartir la cátedra de Escritura y Composición en inglés 2 con el propósito de incorporar en este curso el Centro de escritura. El primer reto era integrar este servicio como parte del curso. Tal integración abarcaba constituir el programa de la materia con contenidos que abarcaran tanto el desarrollo de las habilidades de escritura de los estudiantes tomando el curso como contenidos que les permitieran capacitarse para brindar asesorías de escritura a otros estudiantes escritores. Además, al integrar el Centro de Escritura como parte del curso, se debía encontrar la manera de dar a los estudiantes el crédito por convertirse en asesores de escritura.

El siguiente reto fue difundir la existencia del Centro de escritura. Para tal fin, se utilizó anuncios en reuniones de profesores, volantes de información, visitas a los distintos cursos de la Escuela de idiomas, correos electrónicos y publicaciones en redes sociales. El Centro abrió "sus puertas" (simbólicamente pues no se contaba con una localidad específicamente asignada para este fin, por lo que se inició en el salón de clases asignado para Composición 2) la tercera 
semana del semestre que está compuesto por diecisiete en total, y se mantuvo funcionando hasta la semana catorce del mismo semestre.

Después de las tareas de difusión de la existencia del centro, se presentó el tercero y más desafiante de los retos asumidos al iniciar un Centro de escritura, el cual fue lograr que los estudiantes de los otros cursos vinieran a consultar sobre sus escritos. Esto fue lo más difícil de superar por una razón fundamental, la manera en que la escritura era, y sigue siendo, concebida en la Escuela de Idiomas, a saber, escribir para evaluar en vez de para comunicar. La situación fue que cualquier tarea escrita de todas las asignaturas impartidas era realizada por los estudiantes en el momento de recibir la asignación, en el mismo salón de clases y dentro del tiempo de clases. Este era el escrito evaluado y calificado por los profesores, por lo que resultaba imposible que los estudiantes buscaran ayuda de los asesores de escritura tomando el curso de Composición 2.

A pesar de esto, y gracias a que la autora estaba impartiendo otra cátedra, se solicitó a los estudiantes ir a asesorías, los estudiantes de Didáctica del idioma inglés 1, fueron los primeros en visitar el Centro y ser asesorados. Además, cuatro de los casi treinta profesores que componían la planta docente de la escuela, tuvieron a bien apoyar la labor del Centro asignándoles tareas escritas a sus estudiantes para realizar fuera del salón de clase o entregándoles una revisión con correcciones de un primer borrador y exigiéndoles que asistieran a asesorías en el Centro de escritura para entregar una versión final del mismo. Me encargué personalmente de visitar los salones de clases de estos profesores para programar citas a todos aquellos estudiantes deseosos de visitar el Centro. Este esfuerzo y apoyo de los colegas logró un primer acercamiento a un grupo numeroso de estudiantes que manifestaron sentirse satisfechos de las asesorías recibidas y que gradualmente se convirtieron en visitantes regulares del Centro de escritura.

Al final del semestre, los estudiantes de Composición 2 brindaron más de 100 asesorías a estudiantes en cátedras como: Didáctica del idioma inglés 1, Literatura Británica, Inglés intermedio 1, Inglés Avanzado 1, Gramática del idioma inglés 1 y Métodos y Técnicas de Investigación². Es significativo mencionar, que muchos de los estudiantes que consultaban el Centro cursaban más una de estas materias al mismo tiempo, por lo que consultaban sobre diversas tareas escritas. Además de la diversidad de cátedras por las cuales los estudiantes consultaron, se brindó asesoría en todas las etapas del proceso de escritura. Los asesores apoyaron estudiantes en el proceso previo a empezar a escribir,

Centro de escritura en la Escuela de Idiomas de la Universidad Don 38. Bosco durante la escritura y en los procesos de revisión y edición de escritos. Sin duda

2 Este último curso impartido en español, por lo que los asesores del Centro brindaron asesorías en español también. 
alguna, esta oportunidad se trasladó muy positivamente a las habilidades de escritura de los mismos asesores quienes lograron un mejor entendimiento de la escritura en el contexto académico el cual se vio finalmente reflejado en sus mismos escritos.

\section{La continuidad del Centro de escritura}

El propósito de este artículo ha sido reflexionar, con base en la experiencia obtenida con la implementación de un Centro de escritura, en aspectos cruciales que podrían determinar un cambio significativamente positivo en la preparación de futuros profesionales en las áreas de idiomas de turismo y enseñanza. En primer lugar, es válido cuestionarse como pueden sostenerse actividades de esta naturaleza a través del tiempo si un profesor lo hace como un esfuerzo aislado sin contar con el apoyo y reconocimiento de la institución a la que pertenece (Carlino, 2012, p. 488). Contrario a lo que podrían creerse y después de hacer una investigación con respecto al inicio de programas similares en universidades alrededor del mundo (Molina Natera, 2012; Thaiss, Bräuer, Carlino, Ganobcsik-Williams y Sinha, 2012) se puede concluir que el inicio de un Centro de escritura no ha sido en ningún contexto una tarea fácil. Muchos precursores de Centros de escritura alrededor del mundo han enfrentado diversas dificultades desde falta de presupuesto, como lo ha sido el presente caso, hasta apatía de las partes involucradas. Sin embargo, el hecho de que actualmente existan más de 330 instituciones en seis continentes, de entre los cuales hay siete países latinoamericanos, es evidencia de que la escritura académica está siendo debidamente valorada por muchas instituciones de educación superior alrededor del mundo (Thaiss, Bräuer, Carlino , Ganobcsik-Williams, y Sinha, 2012). Los beneficios reflejados en el aprendizaje, tanto de los asesores como de los escritores, son innegables. Hay una mejor valoración del propósito real de escribir y de las audiencias hacia quienes se escribe, hay mejor entendimiento de la diversidad de textos que se acoplan a los propósitos por los que se escribe y, de manera sobresaliente, hay mejor manejo del conocimiento pues se deja de simplemente recibir y se empieza a transformar el mismo. Considero que sí vale la pena el continuar con este esfuerzo con la confianza de que con constancia los resultados empezaran a manifestarse por sí mismos.

En segundo lugar, es importante renovar los supuestos con respecto a la escritura académica que han permanecido arraigados en los profesores por muchos años. En la medida en que las tareas de escritura sean contextualizadas dentro de las áreas de estudio de cada especialidad y en que se guíe a los estudiantes en el proceso de producir un escrito, se dejara de percibir la escritura como un producto para percibirla como un proceso. Carlino (2003) enfatiza que escribir en la universidad es una actividad realizada "para obtener, analizar, interpretar, 
elaborar y transmitir información" (p. 411). Es decir, la escritura debería ser entendida como el medio para producir información. Tal entendimiento permitirá a los profesores ofrecer apoyo, guía y retroalimentación a los estudiantes durante el proceso. Escribir una tarea dejara de ser una situación remedial de errores y de obtención de una calificación para convertirse en un medio de expresión de ideas y de conocimiento y en una habilidad transferible a las diversas situaciones a las que deberán enfrentarse como profesionales.

\section{Referencias}

Aparna Sinha (Eds.). (2012). Writing Programs Worldwide: Profiles of Academic Writing in Many Places. Perspectives on Writing. Fort Collins, Colorado: The WAC Clearinghouse and Parlor Press. Wac Clearinghouse / Parlor Press pp. $485-498$

Carlino, P. (2003). Alfabetización académica: un cambio necesario, algunas alternativas posibles. Educere 6(20), 409-420.

Carlino, P. (2012) Section essay: who takes care of writing in Latin american and Spanish universities? In Chris Thaiss, Gerd Bräuer, Paula Carlino, Lisa Ganobcsik-Williams, and

Molina Natera, V. (2012). Escritura a través del currículo en Colombia: situación actual y desafíos. magis, Revista Internacional de Investigación en Educación, 5(10).

Thaiss, C. (2012). Origins, Aims, and Uses of Writing Programs Worldwide: Profiles of Academic Writing in Many Places. In Chris Thaiss, Gerd Bräuer, Paula Carlino, Lisa Ganobcsik-Williams and Aparna Sinha (Eds.) (2012). Writing Programs Worldwide: Profiles of Academic Writing in Many Places. Perspectives on Writing. Fort Collins (pp.5-22). Colorado: The WAC Clearinghouse and Parlor Press 